\section{Evoked potential analysis programs for the Lab 8}

\section{GLENN F. WILSON and JAMES GREGORY \\ Wittenberg University, Springfield, Ohio 45501}

Software from Digital Equipment Corporation (DEC) for their Lab 8 (PDP-8/e) computer provides for average response data. However, subsequent analysis of these data is limited to hand measurements of plotted curves or the use of a general-purpose program (i.e., DEC's Daquan). The first method is laborious and error prone. The second is faster and more accurate but yields data which must be further manipulated to arrive at the appropriate voltage and time values. Individual amplifier gains must be included in these adjustments so that comparisons may be made of data from different electrode sites on the same subject, as well as between subjects. To overcome these difficulties, we have written three BASIC language programs to make further analysis of these data easier and more accurate. The PAKCAL program packs the averager output files into single precision, calculates calibration factors for each channel, and calibrates each channel of a file. CRSPLT is used to analyze these files to provide amplitude and latency values of selected points on the averages. These values are output to the terminal in microvolt and millisecond units. A baseline may be derived and the files can be plotted on an X-Y plotter. The AVESUB program averages together groups of averages or subtracts one average from another to form residuals.

Each average of a data file is "calibrated" to permit direct comparisons between different averages from the same subject, as well as between subjects. A file containing $10-$ microV $10-\mathrm{Hz}$ sine-wave averages from each amplifier channel of the recording system is used to obtain a "calibration factor" for each average of the file. The calibration factor is the value that, when multiplied by its sine wave, produces a curve on the X-Y plotter of a standard height, peak to peak. By finding each channel's unique calibration factor, direct amplitude comparisons across channels are possible.

Using PAKCAL, a file containing $10-\mathrm{microV} 10-\mathrm{Hz}$ sine waves (created by a DEC averager) is first packed by removing the low-order words. The calibration factors for each channel are then calculated and saved for use with subsequent data files. These factors may be recalculated at any time by using new sine waves. After each data file is packed and calibrated, it is saved on the mass storage device so that it can be analyzed using the other two programs.

CRSPLT performs the analysis of the calibrated files. Each channel (average) of the data file is individually loaded into memory and displayed on the oscillo- scope. The vertical size of the display may be adjusted using values input from the terminal. This adjustment affects only the display and does not affect subsequent analyses. Two cursors superimposed on the displayed average may be positioned with ADC knobs. Amplitude and latency values of points of interest on the average may be found. Once the cursors are positioned, the microvolt value of points, the microvolt difference between points, and the millisecond latency of each point is printed on the terminal. The procedure may be repeated with new cursor settings.

A baseline at $0 \mathrm{~V}$ can be calculated. This is especially helpful in work with the contingent negative variation (CNV). The zero baseline is calculated by specifying the number of points to be included. The average value of these points is subtracted from all 250 points, thereby adjusting the entire average to its baseline. Another useful feature for CNV research is the ability to average the values of the curve between the cursors and print this value on the terminal. By using this function, one can find the average amplitude of the CNV for a period of several hundred milliseconds preceding the imperative stimulus.

The displayed average may be plotted on an X-Y plotter, with the speed of plotting controlled by an ADC knob. The plot of each average may be repeated to allow for positioning of the $\mathrm{X}$ and $\mathrm{Y}$ offsets. The baseline may aiso be plotted over the average.

A third program, AVESUB, is used to average calibrated files, one channel at a time. A grand average for each condition in an experiment may be calculated. Residuals are provided by subtracting, point for point, one average from another. Deviations from $0 \mathrm{~V}$, either positive or negative, show points where the curves differ in amplitude. The results of both procedures may be plotted on the X-Y plotter or saved on the mass storage device.

Since the programs are written in BASIC, it is relatively easy to modify them or to write new programs to perform other analyses on the packed and calibrated data. For example, CRSPLT has been modified in our laboratory to automatically measure certain points on all averages without having to reposition the cursors for each measurement. The use of BASIC, while having limitations, is generally much faster than developing programs in another language. The programs have been submitted to DECUS for inclusion in their library.

Input. PAKCAL accepts input files from either of the DEC averager programs, Basic Averager or the Advance Averager. The program is currently written to accept files containing 250 data points. Fewer or more data points can be accommodated by modifying the program if sufficient memory is available.

Output. Calibrated files from PAKCAL and grand 
mean files from AVESUB are stored on the mass storage device. Averages are plotted on an X-Y plotter connected in parallel with the oscilloscope. Latency and amplitude values of selected points are printed on the terminal.

Hardware. PDP-8/e with the Lab 8 peripherals, $8 \mathrm{~K}$ of memory, and a mass storage device are the nec- essary hardware. An X-Y plotter is needed to provide hard copies of the averages.

Availability. The programs have been submitted to DECUS, 126 Main Street, Maynard, Massachusetts 01754. Listings and DECtape copies may also be obtained from the first author, Department of Psychology, Wittenberg University, Springfield, Ohio 45501. 